\title{
Mode-selective optical sensing using asymmetric waveguide junctions
}

\author{
Gergely Zsigmond Racz*, Nikos Bamiedakis, Richard Penty \\ Centre for Advanced Photonics and Electronics, Electrical Engineering Division, Cambridge Univeristy, United Kingdom
}

\section{A R T I C L E I N F O}

\section{Article history:}

Received 31 August 2014

Received in revised form 15 April 2015

Accepted 15 April 2015

Available online 14 June 2015

\section{Keywords:}

Planar optical waveguide

Multimode sensing

Mode duplex transmission

Asymmetric junction

\begin{abstract}
A B S T R A C T
Measuring a single analyte in a highly absorptive microfluidic channel has always been a challenge. Even with a highly selective sensing layer, other chemical species can affect the interrogation of the analyte. Matching the evanescent tail with the sensing layer thickness is difficult in case of evanescent field sensing. The tail typically extends beyond the sensing layer, introducing noise and spurious errors in the measurement, which scales up with analyte concentration. In this work therefore, we propose the use of a simple multimode evanescent waveguide sensor that eliminates such common spurious effects. The proposed mode-selective sensing system exploits the sensitivity differences between the different guided modes in detecting the effects of the outer medium in the sensor response. The operation of the sensor device relies on the use of an asymmetric waveguide junction, which enable efficient separation of waveguide modes and therefore detection of their differences in behaviour. The proposed device is shown through simulations to achieve very small estimation errors below $5 \%$, even for very high absorption coefficients of the outer medium of up to 80 times larger than that of the sensing layer.
\end{abstract}

(c) 2015 Elsevier B.V. All rights reserved.

\section{Introduction}

In the last decade, optical sensing has seen a rapid growth as it offers great advantages over other sensing technologies, such as immunity to electromagnetic interference, high selectivity and sensitivity [1]. In particular, lab-on-a-chip optical sensors have attracted considerable interest from the medical industry targeting the development of highly sensitive but low-cost portable devices. As a result, the integration of optical waveguide technologies with sensing elements and microfluidics onto low-cost platforms has received significant attention [2]. Several successful optical detection schemes have been implemented with such integrated waveguide sensors, based on different detection principles such as absorption [3], interferometry [4], spectroscopy [5] and plasmonics [6]. Absorption-based waveguide sensors are the most widely used devices as they are inherently simpler to fabricate and require low-cost light sources and detectors. Such sensors, unlike other common sensing elements such as Mach-Zehnder interferometers, microrings and gratings, do not require the fabrication of complex optical structures, which, in turn, require high-resolution fabrication processes and stringent alignment tolerances in the device

\footnotetext{
* Corresponding author. Tel.: +44 7403039002.

E-mail addresses: gzr20@cam.ac.uk (G.Z. Racz),nb301@cam.ac.uk (N. Bamiedakis), rvp11@cam.ac.uk (R. Penty).
}

assembly and packaging. Recent advances in polymer-based optical materials have enabled the formation of cost-effective multimode waveguides which can be directly integrated onto low-cost printed circuit boards (PCBs) using standard manufacturing and assembly methods of the electronics industry [2]. Recently, a PCB-integrated optical sensor based on the use of multimode polymer waveguides has been demonstrated for ammonia gas sensing. The optical waveguides are functionalised with chemicals dyes, creating a versatile absorption-based sensor that can be directly integrated with the detection electronics onto a low-cost PCB [3].

Despite their simplicity, an important issue regarding the operation of evanescent-wave sensors is the fundamental trade-off between the thickness of the sensing layer and the measurement sensitivity. Relatively thick sensing layers (membranes, typically $\sim 1 \mu \mathrm{m}$ in thickness) exhibit increased sensitivity due to the large overlap of the evanescent field and the sensing area. However, they suffer from slow response and recovery times due to the increased diffusion time of the measurand molecules from the carrier medium (outer medium) into the membrane. In comparison, thinner sensing layers, such as self-assembled monolayers ( $\sim 10-100 \mathrm{~nm}$ in thickness), exhibit very fast responses but can suffer from spurious effects due to the presence of other chemical species in the carrier medium, as the evanescent field extends outside the sensing layer and interacts with these as well as the sensing layer (Fig. 1). It has been shown that such the absorption changes in the outer medium can be as large as 50 times higher than 


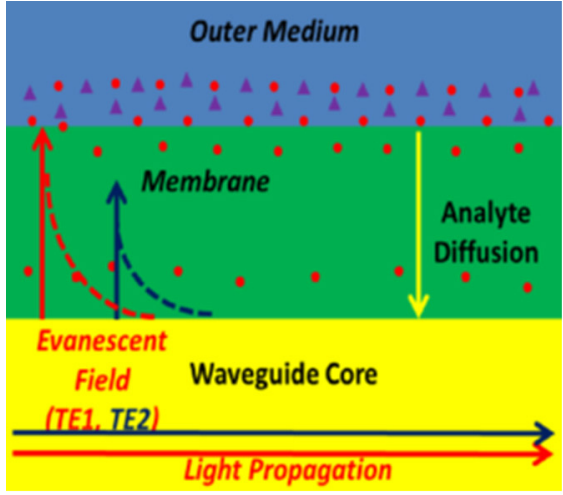

(a)

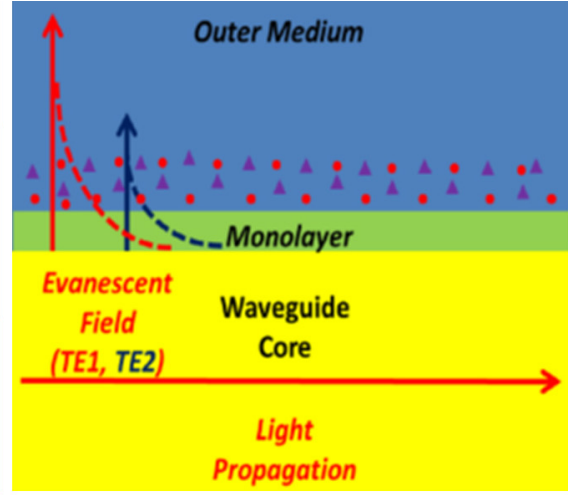

(b)

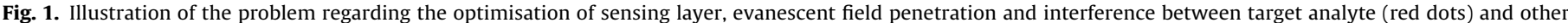

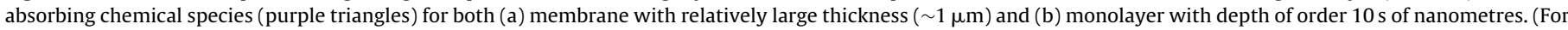
interpretation of the references to colour in this figure legend, the reader is referred to the web version of this article.)

the absorption changes in the sensing layer due to the presence of other (non-specific) chemical species. As a result, serious degradation in the evanescent-wave sensor operation can occur [7]. To overcome this issue, reference waveguides are typically deployed such as in interferometric sensors, in order to distinguish real from spurious sensor response. Here, we propose a novel method of tackling this issue in absorption waveguide sensors using asymmetric mode-selective $Y$-junctions.

Recent developments in data communications towards achieving higher-capacity optical links have created a renewed interest in mode-multiplexed optical waveguide systems, where different modes carry different streams of information [8]. Asymmetric waveguide Y-junctions have been proposed to be used both at the receiver and transmitter end in order to multiplex and demultiplex the different propagating modes and efficiently recover the different data streams transmitted. In the context of optical sensors, we propose for the first time the use of such structures in multimode evanescent-wave absorption sensors in order to overcome the spurious effects due to absorption changes in the outer medium and thus improve their operation and reliability. The proposed sensor device maintains the fundamental simplicity of multimode absorption sensors while overcoming this common issue with such devices. In the sections that follow, the design of the proposed waveguide sensor is presented and its operating principle is described (Sections 2 and 3). Simulation results are reported in Section 4 demonstrating that the device can efficiently detect the analyte of interest with an accuracy better than 95\%, even in the case of a highly absorbing outer medium with an absorption
80 times greater than that of the sensing layer. Finally, Section 5 concludes the paper.

\section{Waveguide sensor design}

The schematic of the proposed absorption-based multimode waveguide sensor is shown in Fig. 2 for a 2-moded interrogation. It comprises of 3 sections: the input coupling section, the sensing section and the asymmetric Y-junction section. Similar devices enabling interrogation of larger number of waveguide modes can be implemented by deploying a larger-order Y-junction and are discussed later. The input section comprises a multimode waveguide which offers higher coupling efficiency and larger alignment tolerances at the device input than the commonly used single mode waveguides. The sensing section comprises the multimode waveguide functionalised with a sensing layer and includes the microfluidic channels that deliver the target analyte to the sensing area. The different waveguide modes propagating in the sensing section have an evanescent tail, which differs in probing depth and extends to a different degree in the sensing layer and outer medium. As a result, different waveguide modes exhibit a different sensitivity to absorption changes in the sensing layer and outer medium. Lower order modes, due to their increased confinement in the waveguide core, exhibit larger sensitivity to changes in the sensing layer, while higher order modes are more sensitive to absorption changes in the outer medium than lower order modes. The asymmetric Y-junction is appropriately designed to separate

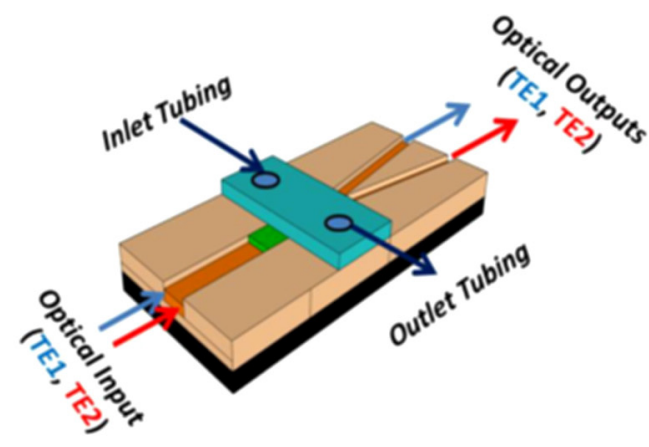

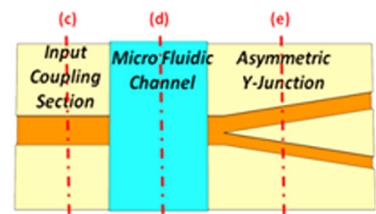

(b)

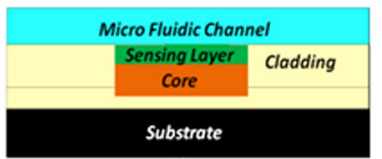

(d)

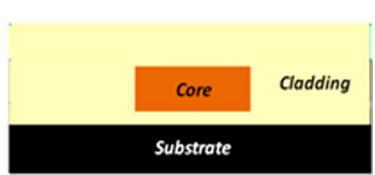

(c)

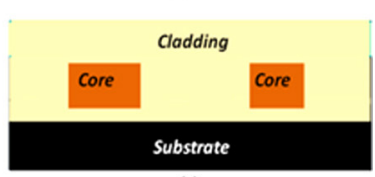

(e)

(a)

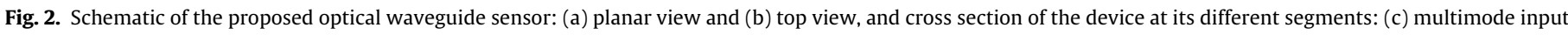
section, (d) sensing section and (e) output section. 


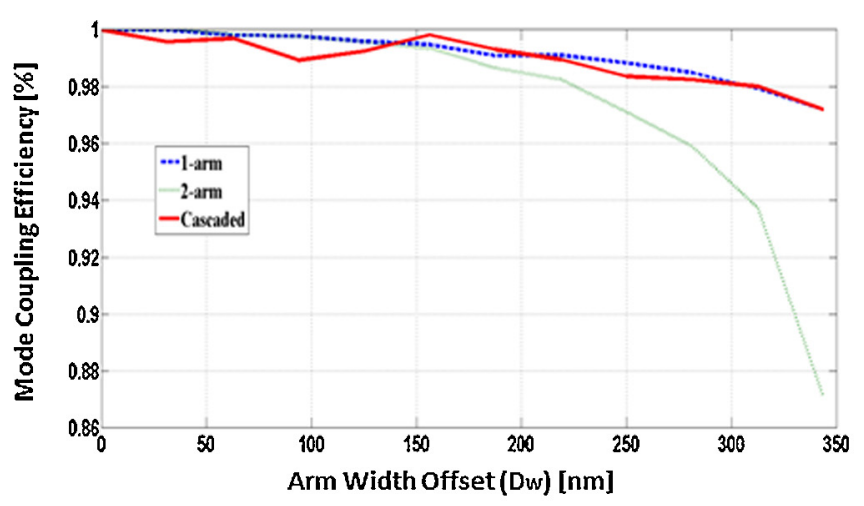

(a)

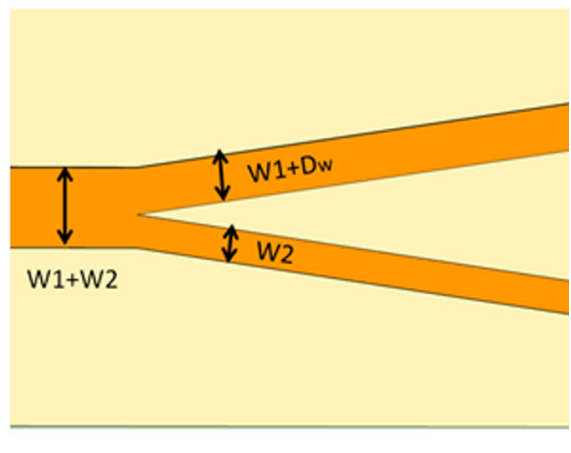

(b)

Fig. 3. Summary of fabrication sensitivity analysis, where mode coupling efficiency changes with arm widths for each device.

the waveguide modes at the corresponding waveguide outputs by matching the effective refractive indices of the guided modes in the transition area [8]. By monitoring the variation of the output power at the different output waveguides, the sensor response due to changes in the sensing layer and the outer medium can be decorrelated.

The number of waveguide modes that are employed in the device can be increased by employing higher-order asymmetric Y-junctions. Such higher-order multimode devices enable the use of larger waveguide dimensions at the device input and can therefore offer improved coupling efficiencies and alignment tolerances and more reliable sensor operation due to the simultaneous monitoring of larger number of interrogating modes. Examples of some higher-order asymmetric Y-junctions are shown in Fig. 3. The design of such high-order mode junctions is a non-trivial process, as it requires precise matching of the effective indices of the waveguide modes in the input multimode section to the output single mode waveguides and an adiabatic transition between the two sections $[8,9]$. Moreover, practical considerations restrict the use of such devices to few-moded junctions. It is shown that the required length for the adiabatic mode transition in the Y-junction dramatically increases with the number of arms, resulting thus in impractically long devices [9], while the required fabrication tolerances for higher-order junctions also become very stringent as the number of arms increases. A practical way however to achieve higher-order devices consists of cascading feasible 2-moded Yjunctions [10]. Fig. 4(c) illustrates a 4-moded device implemented with two stages of 2-moded Y-junctions.

In order to assess the tolerances for the fabrication of such devices, a basic analysis has been carried out. The mode sorting efficiency of a 2-, 3- and a cascaded Y-junction is calculated when the width of one arm of the junction is offset by a small amount $D_{w}$ with respect to its ideal value (Fig. 3). The offset $D_{w}$ is introduced in the smallest arm of each device as its mode coupling performance is more susceptible to the width changes. The results demonstrate that the use of cascaded Y-junctions can enable the formation of higher order mode sorters with more relaxed fabrication tolerances than large asymmetric Y-junctions. The fabrication tolerances of each 2-moded junction can be achieved with standard fabrication methods, while the total length of the device remains practical. As an indication of the required length, the coupling length for a 3-moded junction (Fig. 4b) is found to be $15 \mathrm{~mm}$, while the cascaded 4-moded junction (Fig. 4c) requires a tapered S-bend of only $10 \mathrm{~mm}$ for each two-branched Y-junction. In the simulation studies that follow, the performance of sensor devices employing a 2-moded (Fig. 4a), a 3-moded (Fig. 4b) as well as a cascaded 4moded (Fig. 4c) junction is evaluated. More details on the design process of these junctions can be found in $[9,10]$. By monitoring the variation of the output waveguide modes, the sensor response due to changes in the sensing layer and the outer medium can be de-correlated.

In this study, the performances of all three variants of these structures are evaluated in order to observe how the performance changes with increase number of interrogating modes. The design of such junctions is a non-trivial process, especially in the case of higher-order mode junctions. More details on the design process can be found in [9].

\section{Sensitivity analysis}

The principle operation of the sensor device is described in this section, while a sensitivity analysis is carried out for each variant of the mode-selective sensor. For the purpose of this analysis, the target analyte is assumed to be glucose, as the glucose opto-chemical

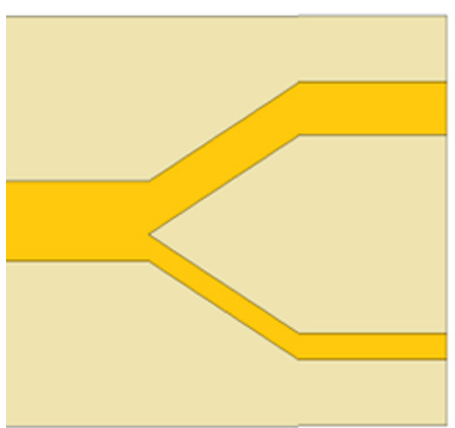

(a)

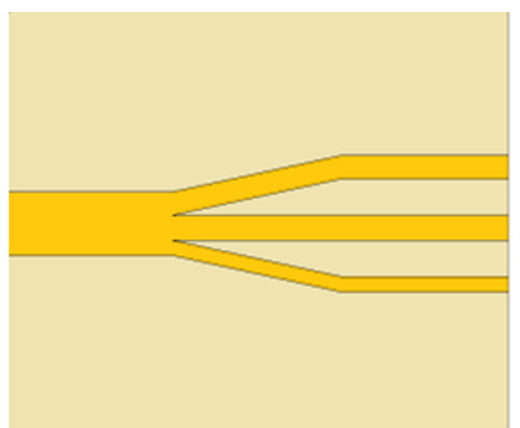

(b)

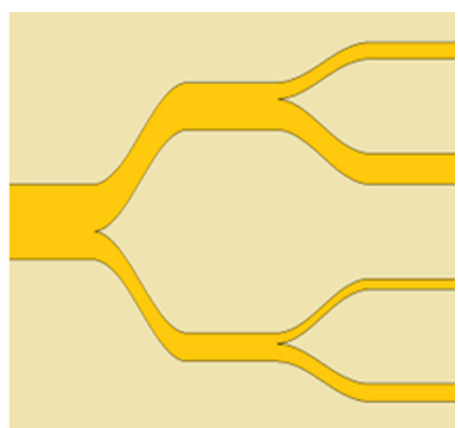

(c)

Fig. 4. Mode sorting Y-junctions for: (a) a 2-moded and (b) a 3-moded device as well as (c) a 4-moded device employing cascaded 2-arm junctions. 


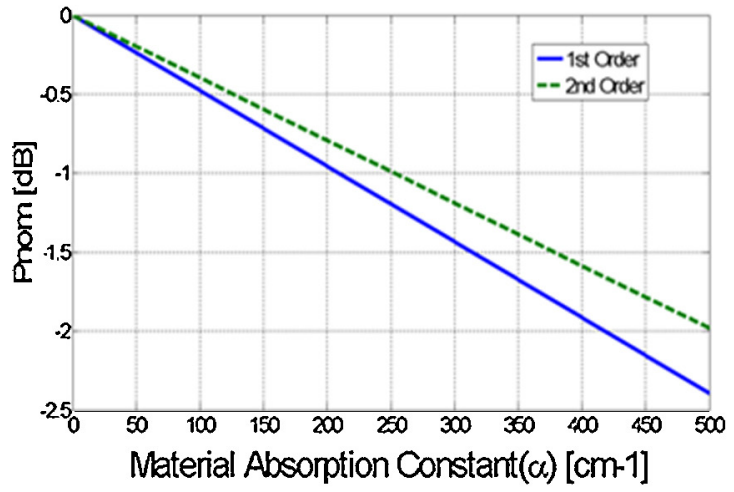

(a)

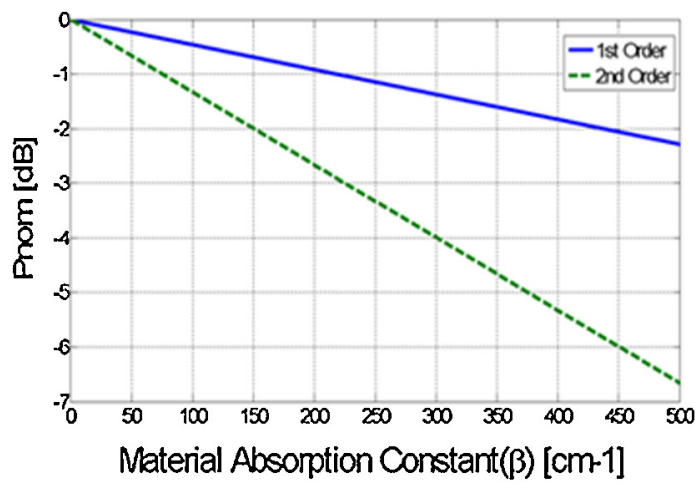

(b)

Fig. 5. Mode sensitivity for absorption changes in (a) the sensing layer (b) the outer medium for each mode.

parameters are well known [12]. In order to study the sensor operation, the electrical field of the waveguide modes needs to be calculated as well as their corresponding absorption coefficients due to changes in the sensing layer and outer medium of the device. The complex effective refractive index of the propagating mode $\left(n_{\text {eff }}\right)$ is given by the expression:

$n_{\text {eff }}=n_{\text {eff }}^{r}+j K$

where $n_{\text {eff }}^{r}$ is the real part of the effective refractive index which depends on the waveguide parameters and light wavelength, while $K$ is the imaginary refractive index that depends on the absorption of the sensing layer and outer medium. The imaginary refractive index $K$ can be evaluated using the absorption coefficient of the glucose at a given wavelength and its concentration based on Eq. (2). A change in analyte concentration translates into changes in the absorption coefficient of the waveguide modes [11]. The molar extinction coefficient $(\varepsilon)$ or molar absorption coefficient $\left(\alpha_{m}\right)$ are used to describe absorption lines or bands and their typical values can be calculated from published data.

$K=0.183 \varepsilon C \lambda=\frac{\alpha_{m} C \lambda}{4 \pi}$

The value of $\varepsilon$ is $14,000 \mathrm{M} / \mathrm{cm}$ at $530 \mathrm{~nm}$ (at $1 \mathrm{mM}$ concentration) for a quinoneimine dye [12]. By using this parameter together with Lambert-Beer's law, the material absorption coefficient $\left(\alpha_{m}\right)$ can be found for different concentrations $(C)$ of glucose. Both the real $\left(n_{\text {eff }}^{r}\right)$ and imaginary part $(K)$ of the complex modal refractive index can change as a function of glucose concentration, but the deviation in magnitude of $n_{\text {eff }}^{r}$ is negligible compared to $K$ [11]. The analyte concentration employed in these studies ranges between 0 and $16 \mathrm{mM}$ of glucose, which translates to a material absorption coefficient $\left(\alpha_{m}\right)$ between 0 and $500 \mathrm{~cm}^{-1}$.

A matching waveguide model is produced in Fimmwave/Fimmprop for each of the studied sensor devices (structures in Fig. $4 a-c$ ). The waveguide core and cladding refractive index is assumed to be 1.510 and 1.513 respectively at $530 \mathrm{~nm}$, matching siloxane polymer materials [2]. The sensing section length is kept constant at $5 \mathrm{~mm}$ for all the simulations, while the sensing layer thickness is chosen to be $200 \mathrm{~nm}$, unless stated otherwise. The refractive index of the outer medium is 1.33 (water-based solution), while that of the sensing layer is 1.45 (typical for a protein recognition layer). The penetration depth of the evanescent field of the 2 nd order mode in the sensing section is found to be $\sim 0.5 \mu \mathrm{m}$ due the small refractive index difference $\Delta n$ of $3 \times 10^{-3}$ between core and cladding materials. The absorption of the sensing layer $\alpha$ depends on the glucose concentration $C$ and can be determined from Eq. (2), while the outer medium is assumed to have an additional absorption coefficient $\beta$ that can be varied between 0 and $100 \times \alpha$. The case $\beta=0$ represents the ideal case where the outer medium does not exhibit any spurious absorption at the wavelength of interest and, therefore does not interfere with the sensor operation. On the other hand, the case $\beta=100 \times \alpha$ corresponds to a "worst-case" scenario which can cause serious interference with the analyte concentration estimation. For each device studied, the waveguide modes are found using the Fimmwave mode solver and their corresponding propagation loss due to the absorption in the two layers is derived for the different values of $\alpha$ and $\beta$.

Fig. 5 shows the variation of the output power for the two output arms of a 2-moded sensor as the function of the absorption coefficients of the sensing layer $(\alpha)$ and the outer medium $(\beta)$. It can be seen that the lower order mode is slightly more sensitive to absorption changes in the sensing layer (larger slope) whereas the 2nd order mode is much more sensitive to the absorption changes in the outer medium.

From the above power variation plots, it can be observed that the power of each mode depends linearly (in logarithmic scales) on the absorption coefficients $\alpha$ and $\beta$ of the sensing layer and outer medium respectively. As a result, the power of each waveguide mode at the sensor output can be easily expressed with simultaneous equations. Using the asymmetric waveguide junction, the optical power of each waveguide mode can be efficiently monitored, while the optical loss of each mode can be expressed as:

$\left[\begin{array}{l}A_{1} \\ A_{2}\end{array}\right]=\left[\begin{array}{ll}S_{11} & S_{12} \\ S_{21} & S_{22}\end{array}\right]\left[\begin{array}{l}\alpha \\ \beta\end{array}\right]$

where $A_{i}$ is the total loss (in $\mathrm{dB}$ ) of mode $i$, and $S_{1 i}$ and $S_{2 i}$ are the absorption coefficients of mode $i$ to absorption changes in the sensing layer and the outer medium respectively. By measuring the power of each waveguide mode before and after the insertion of the analyte, the mode loss $A_{i}$ can be found and therefore, by solving Eq. (4), we can obtain the unknown absorption coefficients $\alpha$ and $\beta$ of a particular sample by finding the least-squares solution of the equation:

$$
\left[\begin{array}{l}
\hat{a} \\
\hat{b}
\end{array}\right]=\left[\begin{array}{ll}
S_{11} & S_{12} \\
S_{21} & S_{22}
\end{array}\right]^{-1}\left[\begin{array}{l}
A_{1} \\
A_{2}
\end{array}\right]
$$

The described analysis can be extended for higher-order sensor devices. The increase in the number of interrogating modes can further improve the detection process by increasing the size of the sensitivity matrix S. Eqs. (5) and (6) show the equations to be solved 


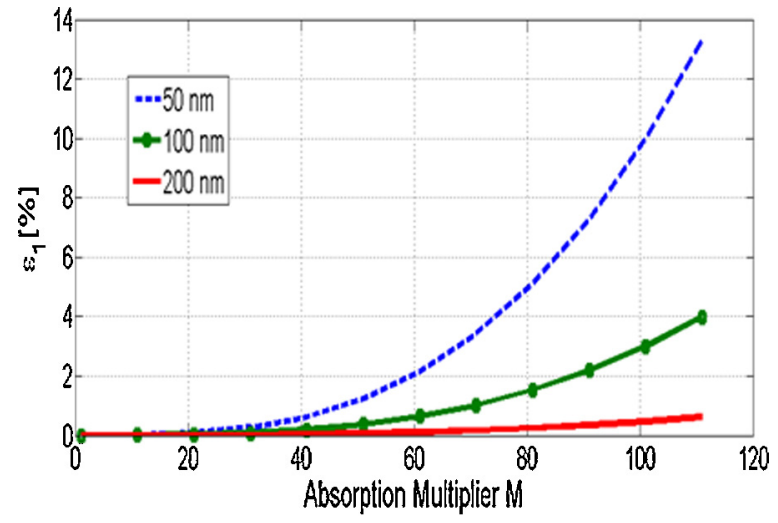

Fig. 6. Relative error in the estimation of the sensing layer absorption $\alpha$ obtained with the proposed mode-selective sensor as a function of the spurious absorption of the outer medium for a range of sensing layer thicknesses.

for the 3 and 4-moded sensors. The least-square solution for Eqs. (4)-(6) can be easily derived with common linear algebra methods.

$$
\begin{aligned}
& {\left[\begin{array}{ll}
S_{11} & S_{12} \\
S_{12} & S_{22} \\
S_{31} & S_{32}
\end{array}\right]\left[\begin{array}{l}
\hat{a} \\
\hat{b}
\end{array}\right]=\left[\begin{array}{l}
A_{1} \\
A_{2}
\end{array}\right]} \\
& {\left[\begin{array}{l}
S_{11} S_{12} \\
S_{21} S_{22} \\
S_{31} S_{32} \\
S_{41} S_{42}
\end{array}\right]\left[\begin{array}{l}
\hat{a} \\
\hat{b}
\end{array}\right]=\left[\begin{array}{l}
A_{1} \\
A_{2}
\end{array}\right]}
\end{aligned}
$$

\section{Simulation results}

In order to evaluate the accuracy of the estimation method, the simulation model is run for different values of $\alpha$ and $\beta$ for the three devices. The concentration of glucose at the sensing layer is assumed to be constant at $1.5 \mathrm{mM} / \mathrm{l}$, while the thickness of the sensing layer is varied between 50 and $200 \mathrm{~nm}$. The outer medium absorption $\beta$ is assumed to be a multiple $M$ of the sensing layer absorption coefficient $\alpha$, ranging from $M=0$ which represents the ideal case of a non-absorbing carrier, to $M=110$ which represents the extreme case of a very highly absorbing medium. For such a highly absorbing outer medium the spurious response of the sensor is expected to be very large. The simulation is carried out for each sensor device for each combination of the $(\alpha, \beta)$ parameters and the corresponding mode loss values $A_{1}$ and $A_{2}$ for each combination of $(\alpha, \beta)$ are obtained from the simulations.

$\beta=M * \alpha$

The obtained $A_{1}$ and $A_{2}$ values are fed into the sensor analysis method described above, providing the estimated $\hat{a}$ and $\hat{b}$ values for the sensing layer and outer medium respectively. Fig. 5 shows the relative error $\varepsilon_{1}$ in the estimation of actual values of $\alpha$ obtained with the model for the 2-moded sensor device for the different values of the absorption multiplier $(M)$ :

$\varepsilon_{1}=\left|1-\frac{\hat{a}}{\alpha}\right|$

As shown in Fig. 6, the estimation error is very low, which means the estimated values $\hat{a}$ and $\hat{b}$ are very close to the real value of $\alpha$ and $\beta$ for the all thicknesses studied. The relative error for $\alpha$ is below $2 \%$ even in the case of a highly absorptive outer medium with an absorption coefficient 80 times larger than that of the sensing layer with thicknesses larger than $100 \mathrm{~nm}$. For a thinner sensing layer, such as in a self-assembled monolayer ( $\sim 10 \mathrm{~s}$ of nanometers), a slightly larger error of $5 \%$ is obtained up to an $M$ value of 80 due to the increased effect of the outer medium. Overall, the simulation results indicate a small estimation error for all the thicknesses studied, demonstrating the immunity of the proposed sensor to spurious response due to absorption changes in the outer medium, even in the extreme case of presence of highly absorbing species.

For comparison, the operation of the multimode absorption sensor device without the mode-sorting junction is also studied. The structure of such a device is shown in Fig. 7 and it comprises only the input, output and sensing waveguide sections. For such a device, only the total power at the output of the device can be monitored due to the absence of the mode-sorting junction. As a result, the effect of the outer medium on the measurement cannot be distinguished. The absorption of the sensing layer a hat is estimated based only on the absorption coefficients $S_{1 i}$ of each waveguide mode to the changes in the sensing layer. For a two-moded device

$A_{\text {tot }}=A_{1}+A_{2}=S_{11} * \hat{a}+S_{12} * \hat{a}$

Fig. 7a shows the error in the estimation of the absorption coefficient a due to the absorption of the outer medium. The plot indicates a much larger error in the absorption estimation demonstrating

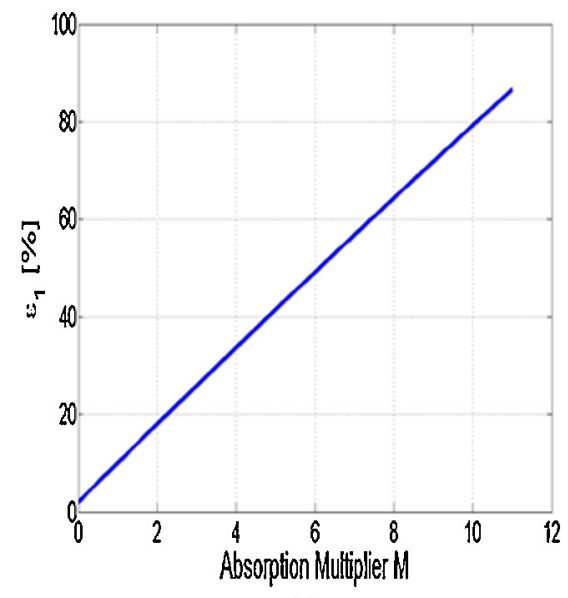

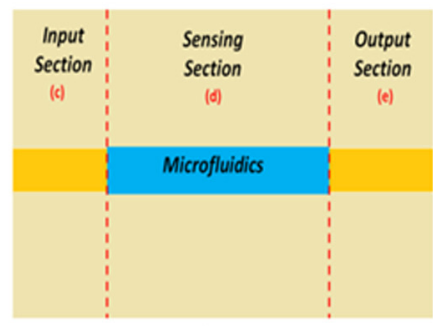

(b)

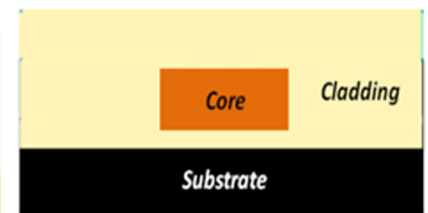

(c,e)

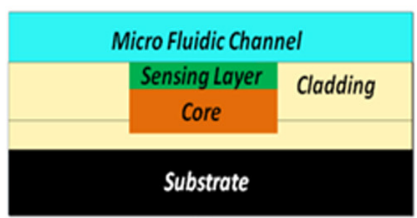

(d)

(a)

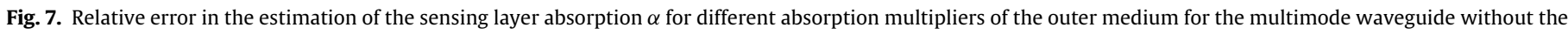
use of asymmetric junctions (structure (b) and cross section (c, d)). 


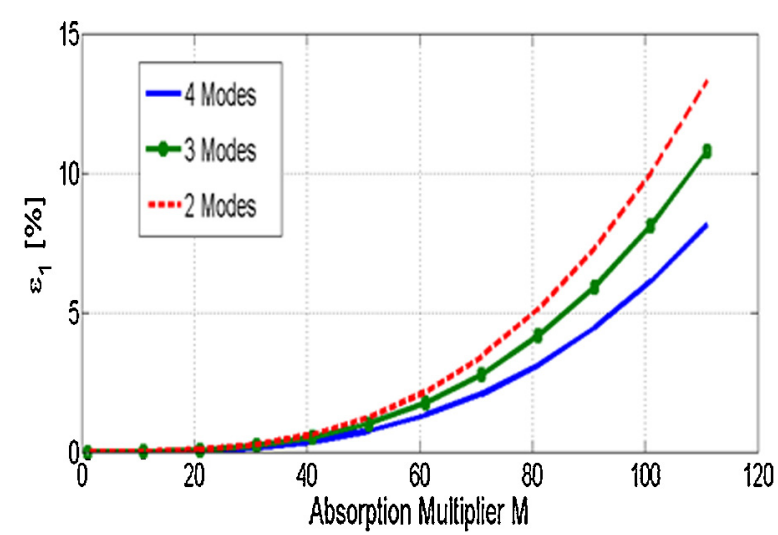

Fig. 8. Relative error in the estimation of the sensing layer absorption $\alpha$ for different absorption multipliers of the outer medium for the three different mode-selective sensors.

the benefits of the use of the mode-sorting junction in the sensor device.

An error estimation analysis similar to the one carried out for the 2-moded sensor, is performed for the 3 and 4 -moded sensor devices shown in Fig. 4. It is expected that the use of larger sensitivity matrices in the absorption estimation [Eqs. (5) and (6)] should provide an improved sensor performance for a larger number of interrogating modes. Fig. 8 shows the relative error in the estimation of the absorption coefficient $\alpha$ as a function of absorption multiplier $M$ for the different number of interrogating modes in the device. As expected, the higher-order devices offer an improved performance. The error in the concentration estimation drops from $10 \%$ to $6 \%$ for $M=100$ (highly absorbing outer medium), as the number of interrogating modes was increased from 2 to 4 . It has to be noted however, that if the spurious absorption of the outer medium does not exceed $\sim 40$ times the absorption change in the sensing layer, similar quality low-error detection can be achieved with the 2-moded device which is simpler to fabricate.

Finally, the sensor enables the estimation of the material absorption coefficient $\hat{b}$ of the outer medium following the same process. The error in the outer medium concentration estimation can be described as:

$\varepsilon_{2}=\left|1-\frac{\hat{b}}{\beta}\right|$

It can be noticed (Fig. 9) that the estimation of the outer medium absorption is very accurate even for large absorption multipliers, while similar good results are obtained from all types of devices.

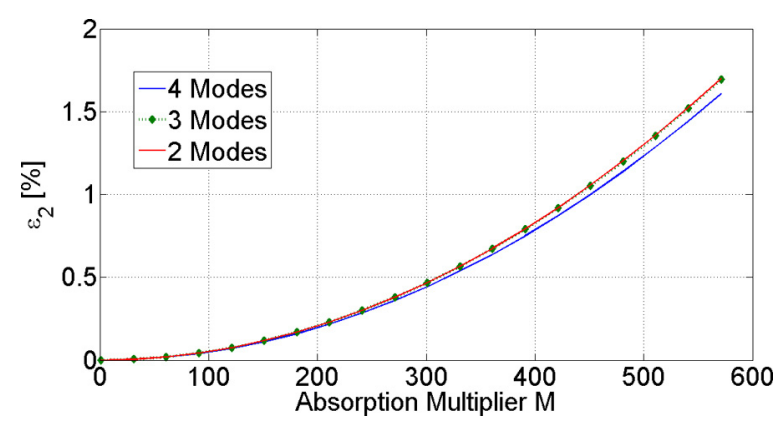

Fig. 9. Relative error in the estimation of the outer medium absorption $\beta$ obtained with the proposed mode-selective sensor for a different number of interrogating modes in the device.

\section{Conclusion}

In this paper, the use of a novel multimode evanescent-wave absorption sensor based asymmetric Y-junctions is proposed. The sensing layer is interrogated by more than one waveguide mode, which can then be separated to different waveguide output arms using an appropriately designed asymmetric Y-junction. The device offers immunity to the common problem of evanescent-wave absorption sensors due to the spurious absorption of the outer medium. By monitoring the behaviour of the different waveguide modes, it enables de-correlation of the real from the spurious absorption. Simulation studies are carried out on the basic waveguide sensor demonstrating that the device can accurately estimate the absorption changes in the sensing layer, even in the extreme case of a highly absorbing outer medium. Very small estimation errors below $5 \%$ are obtained for absorption coefficients of the outer medium which are up to 80 times larger than that of the sensing layer. The sensor performance is compared to the performance obtained from a reference absorption waveguide sensor without the asymmetric waveguide junction demonstrating the benefits brought by the proposed sensor structure. The detection accuracy is improved for higher-order sensor devices due to the larger number of monitored waveguide modes but at the increased cost of device length and reduced fabrication tolerances. The proposed devices have a strong potential for use in chemical, environmental and bio-medical sensing applications.

\section{Acknowledgement}

This work is funded by EPSRC UK.

\section{References}

[1] A.L. Washburn, R.C. Bailey, Photonics-on-a-chip: recent advances in integrated waveguides as enabling detection elements for real-world, lab-on-chip biosensing applications, Analyst 136 (2011) 227-236.

[2] R.A. Potyrailo, S.E. Hobbs, G.M. Hieftje, Optical waveguide sensors in analytical chemistry: today's instrumentation, applications and trends for future development, Fresenius', J. Anal. Chem. 362 (1998) 349-373.

[3] N. Bamiedakis, T. Hutter, R.V. Penty, I.H. White, S.R. Elliott, PCB-integrated optical waveguide sensors: an ammonia gas sensor, J. Lightw. Technol. 31 (10) (2013) 1628-1635

[4] B.J. Luff, J.S. Wilkinson, J. Piehler, U. Hollenbach, J. Ingenhoff, N. Fabricius Integrated optical Mach-Zehnder biosensor, J. Lightw. Technol. 16 (4) (1998) 583-589.

[5] M. Ramuz, D. Leuenberger, L. Bürgi, Optical biosensors based on integrated polymer light source and polymer photodiode, J. Polym. Sci. B: Polym. Phys. 49 (2010) 80-87.

[6] T.M. Chinowsky, Portable 24-analyte surface plasmon resonance instruments for rapid, versatile biodetection, Biosens. Bioelectron. 22 (2007) 2268-2275.

[7] T. Koster, P. Lambeck, An integrated optical platform for absorptive sensing of chemical concentrations using chemo optical monolayers, Meas. Sci. Technol. 13 (2002) 1230-1238

[8] J.D. Love, A. Ankiewicz, Purely geometrical coarse wavelength multiplexer/demultiplexer, Electron. Lett. 39 (2003) 1385-1386.

[9] N. Riesen, J.D. Love, Design of mode-sorting asymmetric Y-junctions, Appl. Opt. 51 (15) (2012) 2778-2783.

[10] A.M. Bratkovskya, J.B. Khurgin, E. Ponizovskayaa, W.V. Sorina, M.R.T. Tana, Mode division multiplexed (MDM) waveguide link scheme with cascaded Yjunctions, Opt. Commun. 309 (2013) 85-89.

[11] G. Stewart, B. Culshaw, Optical waveguide modelling and design for evanescent field chemical sensors, Opt. Quantum Electron. 26 (1993) S249-S259.

[12] U.S. Kayyali, T.B. Moore, J.C. Randall, R.J. Richardson, Neurotoxic esterase (NTE) assay: optimized conditions based on detergent-induced shifts in the phenol/4aminoantipyrine chromophore spectrum, J. Anal. Toxicol. (1991) 86-89.

\section{Biographies}

Gergely Zs. Racz received his Master degree in Electrical and Electrical Engineering from University College London in 2011. He is currently pursuing his PhD at University of Cambridge as part of the Doctorial Training Programme in Photonic Systems Development. His research is focused on integrated photonic system development for sensing applications. At the moment he is serving president of the Hungarian Student Association in the United Kingdom. Area of research he is interested 
include integrated optical sensors, point-of-care diagnostics and high speed optical modulators.

Nikos Bamiedakis received his Diploma in Electrical and Computer Engineering from the National Technical University of Athens (NTUA), Greece in 2003 and PhD in Engineering from the University of Cambridge, Cambridge, UK in 2008. Since then, he has been working as a Research Associate at the Centre for Advanced Electronics and Photonics (CAPE) at the Engineering Department, University of Cambridge. His research focuses on board-level optical interconnects, low-cost optical sensors and visible light communication systems.
Richard Penty graduated from the University of Cambridge with a degree in Engineering and Electrical Sciences in 1986 and a PhD in Photonics in 1990. In 1996 he moved to the University of Bristol as a lecturer in electrical and electronic engineering subsequently being promoted to Reader and Professor of Photonics. In 2001 he moved to the Cambridge University Engineering Department and in 2013 he was elected as the master of Sidney Sussex College. His research interests include photonic integration, optical data communications, MMF systems (digital and analogue), high-speed optical communications systems, and distributing sensing using RFID. 